\section{PSicología IBEROAMERICANA}

\section{Psicología Iberoamericana}

ISSN: 1405-0943

psicología.iberoamericana@uia.mx

Universidad Iberoamericana, Ciudad de

México

México

Mercado Maya, Aída; Oudhof van Barneveld, Hans; Robles Estrada, Erika Visión existencial de la depresión en adultos mayores del Valle de Toluca, Estado de México

Psicología Iberoamericana, vol. 22, núm. 2, julio-diciembre, 2014, pp. 64-71

Universidad Iberoamericana, Ciudad de México

Distrito Federal, México

Disponible en: http://www.redalyc.org/articulo.oa?id=133938134008

- Cómo citar el artículo

Número completo

- Más información del artículo

- Página de la revista en redalyc.org

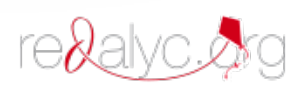

Sistema de Información Científica

Red de Revistas Científicas de América Latina, el Caribe, España y Portugal Proyecto académico sin fines de lucro, desarrollado bajo la iniciativa de acceso abierto 


\title{
Visión existencial de la depresión en adultos mayores del Valle de Toluca, Estado de México
}

\author{
Existential Perspective on Depression among the Elderly \\ in the Valley of Toluca, Mexico
}

\author{
Aída Mercado Maya \\ Hans Oudhof van Barneveld \\ Erika Robles Estrada \\ Universidad Autónoma del Estado de México \\ Facultad de Ciencias de la Conducta
}

\section{RESUMEN}

La depresión constituye un problema de salud en población gerontológica, ya que incide en el funcionamiento físico y emocional de la persona. Ésta puede pasarse por alto -porque los adultos mayores muestran síntomas menos evidentes de pena o tristeza- o asociarse con los cambios propios de esta edad. Desde la perspectiva existencial, fundamento teórico de este estudio, constituye la pérdida de valor y del sentido de la vida. Se trabajó con 690 adultos mayores, de ambos sexos, entre los 60 y 70 años de edad, residentes en el Valle de Toluca, Estado de México. Se aplicó la Escala de Automedición de la Depresión de Zung, con un diseño de investigación no experimental transversal descriptivo. El análisis de los datos se efectuó a partir de la t de Student para muestras independientes y el análisis de varianza de una vía. Los resultados mostraron diferencias significativas respecto al sexo, edad, condición de retiro, actividad física y condición de salud.

Palabras clave: análisis existencial, depresión, adulto mayor, vejez, envejecimiento.

\section{ABSTRACT}

Depression is a health problem among the elderly, since it affects the individual's physical and emotional state. It can be overlooked because signs of sadness or grief are less apparent among the elderly, or are associated with changes inherent in people of this age. From an existential perspective, which is the theoretical basis for this study, we examine how people ascribe less importance to heir lives, and question their meaning. The study population consisted of 690 old-aged people, of both sexes, between 60 and 70 years old, resident in the Valley of Toluca, Mexico. The Zung Self-Rating Anxiety Scale was applied, with a non-experimental cross-sectional-descriptive research design. The data analysis was carried out on the basis of the Student t for independent samples, and the one-way analysis of variance. The results showed significant differences in terms of sex, age, retirement situation, physical activity and health condition.

Keywords: Existential Analysis, Depression, Elderly, Old Age, Ageing. 


\section{INTRODUCCIÓN}

El Instituto Nacional de Estadística y Geografía (INEGI) identificó en el 2010 que la población mayor de 60 años en México superó los 10.9 millones de habitantes, lo que representó a más de $9.3 \%$ de la población total. Así, el envejecimiento es un componente característico de la dinámica de población y un sello de los tiempos modernos, de tal forma que envejece el individuo y envejece la sociedad. Pederzini (2010) define el envejecimiento poblacional como: “(...) un proceso gradual en el que la proporción de adultos y ancianos aumentan en una población, mientras disminuye la proporción de niños y adolescentes" (p. 35).

Aunado a lo anterior, el Consejo Nacional de Evaluación de la Política de Desarrollo Social (Coneval, 2012), refiere que en México 43\% de los adultos mayores se encuentra en situación de pobreza multidimensional, entendida como la situación de una persona cuando carece de al menos uno de sus derechos relacionados con el desarrollo social y sus ingresos son insuficientes para adquirir los bienes y servicios que requiere para satisfacer sus necesidades. En la información recabada en la Encuesta Nacional sobre Salud y Envejecimiento en México 2012 (Enasem, 2012) se identifica que en los adultos mayores participantes predomina la percepción de un estado deficiente de salud, esto es, de regular a mala, con $57.1 \%$ de los hombres y $67.5 \%$ de las mujeres. Respecto a la autonomía para realizar actividades de la vida diaria, las mujeres admiten con mayor frecuencia tener dificultad para ejecutar seis tareas cotidianas: vestirse, acostarse y levantarse, caminar, usar el baño, bañarse y comer, lo cual las coloca en una situación de mayor vulnerabilidad.

$\mathrm{Al}$ respecto, Blazer (2003) afirma que con el aumento de los adultos mayores, se espera una mayor incidencia de múltiples enfermedades crónicas, entre las que se incluye la depresión. Es probable que esta última constituya la principal causa de sufrimiento y mala calidad de vida durante la vejez, ya que junto con los síntomas depresivos, producen un efecto negativo en las capacidades funcionales del adulto mayor, al incidir en sus capacidades físicas y sociales (Ávila, Melano, Payette \& Amieva, 2007).

Lo anterior plantea un problema social de primer orden, ya que las personas mayores como grupo diferente e importante en el ámbito demográfico, presenta rasgos culturales específicos e intereses propios, requiere que la sociedad garantice el incremento de su bienestar y el desempeño de roles que les permitan una existencia plena y con sentido, además del desarrollo de actitudes que pongan fin a la marginalidad y al desarraigo, a la inseguridad, a la falta de calidad de vida y a todos los prejuicios, mitos y estereotipos que forman parte del imaginario social y que envuelven el envejecimiento.

Salvarezza (2005) afirma que sólo conociendo este proceso se podrá preparar y educar para afrontar dicha etapa de manera adaptativa y sana. Lo cual no constituye un proceso simple o unitario, sino una serie de aspectos ligados entre sí, aunque no necesariamente sincrónicos y que, a su vez, se asocian con la edad cronológica, pero sin coincidir con ella. El envejecimiento se despliega en una secuencia compleja de cambios que tienen lugar en distintos niveles: biológico, psicológico y social. Asociados a una disminución de la viabilidad, son de carácter universal, progresivo, decremental e intrínseco. Si bien este conjunto de fenómenos ocurre en todos los organismos vivos, no se envejece de igual manera, ni cada parte del organismo envejece al mismo tiempo.

El envejecimiento, como todo lo humano, siempre lleva el sello de lo singular, lo único, lo individual, por tanto, cada persona envejece en una forma distinta y en este proceso intervienen diversos factores de carácter hereditario, de sexo, estilo de vida y relaciones afectivas, por lo que el envejecimiento es una especie de reacción en cadena, en la que un cambio adverso provoca otro. Se trata de una expresión del estilo de vida, de la actitud hacia la vida y hacia los demás, lo cual se acompaña de la respuesta afectiva de la persona frente a los avatares de la vejez. Arriola (2000), sostiene que aproximadamente a los sesenta años se inicia la etapa de la vejez, cuando se presenta un declive gradual del funcionamiento de todos los sistemas corporales. Envejecer es un proceso dinámico, gradual, natural e inevitable, en el que se dan cambios a escala biológica, corporal, psicológica y social.

De acuerdo con Mishara y Riedel (2000), los cambios fisiológicos que se producen a partir de la sexta década de la vida comportan una complejidad. Éstos pueden predisponer la aparición de varias enfermedades en forma simultánea. Al presentarse juntas dichas 
dolencias, se potencian entre sí y, además, enmascaran el curso natural de cada una de ellas, ya que se desfiguran los síntomas y signos individuales característicos.

Los datos reportados por el INEGi (2010) muestran que las mujeres viven más años en promedio que los hombres; en el 2010 la esperanza de vida fue de 77 años para mujeres y 71 para los hombres; en 2014, se espera en poco más de 77 años para las mujeres, y en 72 años para los hombres. Al respecto, Ortiz de la Huerta (2004) refiere que las mujeres viven tres o cuatro años más que los hombres, por lo cual no se mueren de vejez, sino por las enfermedades que acompañan a ésta. Este grupo, por tanto, presenta menor mortalidad, pero mayor morbilidad, es decir, las mujeres se enferman más, pero no para morirse, sino para durar mucho tiempo con las enfermedades, que son crónico-degenerativas. Mientras que Gallo y Kasses (2009), puntualizan:

El pasaje a la vejez presenta una situación simétricamente opuesta de desesperanza, pues conlleva perder posiciones alcanzadas con tanto esfuerzo, para retroceder en el estatus social, abandonando roles productivos e incorporándose a la población pasiva. El individuo ya no elige su futuro, sino que se prepara para su muerte, primero social y más adelante, física. En esta difícil crisis vital, el individuo debe preparase para asumir la irrenunciable responsabilidad de darle una respuesta con sentido a esta nueva invitación a la vida que significa la propuesta de una nueva edad. Este desafío no es fácil, pues se está inmerso en una cultura que sobrevalora la vida plena, la juventud, la sexualidad genital y orgásmica, la rapidez y la productividad (pp. 10-14).

La pérdida de las habilidades funcionales normales conlleva que tanto la persona como los roles y tareas definidos socialmente se vean afectados, lo cual implica que el nivel de socialización del adulto disminuya, dado que las limitaciones en su salud pueden perjudicar el contacto con otros (Peláez, 2005). La pérdida de la función social es en particular complicada, puesto que los roles sociales son, por lo general, fuentes de pertenencia, dinamismo y valoración. Al respecto, Iglesias (2001) plantea que el retiro laboral puede desembocar en el aislamiento social. Además, cuando se tiene una situación económica precaria y mala salud, la jubilación constituirá una carga difícil de superar (García, 2003).

$\mathrm{Al}$ respecto, Frankl (2001) explica que cuando el hombre se niega a sí mismo, vive a merced de la facticidad de su destino, dominado por aquello que compete al plano psicofísico, no puede distanciarse de sí mismo, pues se llena de vacío, desesperación y de sufrimiento innecesario, al no poder articular su capacidad de oposición frente a los condicionamientos no sólo biológicos, sino también psicológicos o sociales; por tanto, se encuentra determinado por dichos condicionamientos, que se asocian con alguna enfermedad. Vinculado a lo anterior, se debe considerar el efecto de devaluación que producen los estereotipos negativos creados por la sociedad respecto a la vejez y que los adultos mayores, producto de esa misma sociedad, han ido asimilando en etapas previas de su vida. Esta tendencia a la marginalización, rechazo, temor, desagrado, negación, agresión, actitudes todas ligadas entre sí, opera discriminando a la persona que envejece. La falta de comunicación de afectividad y la incomprensión son factores determinantes y creadores de tristeza y de enfermedades (Salvarezza, 2005).

De manera equivocada, la sociedad valora todo aquello que resulta productivo, por lo que fácilmente se considera que las personas mayores no aportan nada, o por el contrario, que representan una carga para la sociedad. En consecuencia, se hace una valoración negativa y poco respetuosa que puede derivar en malestar y pérdida de la ilusión, alegría y ánimo, abandono de las redes de relación y de sí mismo, falta de un objetivo en la vida, así como tristeza, desesperanza y la soledad, que coloca a los mayores como sujetos pasivos que subsisten entre recuerdos y nostalgias de tonalidad depresiva. Dicho tono afectivo merma la calidad de la vivencia de estos años, ya que la depresión constituye una información afectiva de la pérdida de valor y de sentido, en la que inciden aspectos biológicos, psíquicos y sociales. El sentido existencial de los estados depresivos radica en un debilitamiento de la relación del individuo con su propia vida.

Desde el análisis existencial, la depresión es un disturbio que resulta agobiante porque afecta la vivencia de los valores. Todas sus manifestaciones se pueden considerar como una lucha de la persona por percibir el 
valor de su propia vida. Esto significa, de acuerdo con Frankl (2001), que no se concibe sólo como un disturbio psíquico con una mayor o menor participación somática, sino también como un disturbio que afecta tanto las disposiciones como las actitudes, las decisiones y la vivencia espiritual del mundo y de sí mismo. Längle (2004) explica que lo específico en el trastorno de carácter depresivo es la percepción del valor en el complejo relacional. Es decir, aquellas cosas que normalmente alegraban o que resultaban agradables o interesantes para la persona, palidecen y pierden su color, fenómeno que es conocido como afección negativa, la cual implica una pauperización de la nutrición espiritual, que representa los valores para la persona, ya que éstos son el contenido que llena la propia vida y que mantiene y hace atractivas las relaciones. Su importancia existencial radica en la pérdida de la vivencia del valor, ya que los valores tienen un efecto vivificador, fortalecedor y nutritivo en la persona y la psique del hombre.

En palabras de Fizzotti (2006), los cambios en el estado de ánimo suelen derivarse del sentimiento de falta de sentido íntimamente vinculado a un sentimiento de vacío interior, conocido como vacío existencial, y que precede a la frustración existencial; este vacío se manifiesta cuando la persona ha perdido el para qué de su existencia, es decir, su sentido. La pérdida de valor acontece, por tanto, primariamente en los sentimientos y conduce a la perturbación de la vivencia y de la disposición anímica, que son características de la depresión.

Acevedo y Battafarano (2003) plantean que si el hombre es un ser en el mundo, éste incluye un mundo del sentido y de los valores. El sentido y los valores son las razones que mueven al ser humano a adoptar un determinado comportamiento, son el logo que la psique busca al trascenderse a sí misma. Lo anterior no es posible en el caso de la depresión, ya que ésta es un reflejo de la percepción y vivencias negativas que matizan las cualidades de la vida. Para Frankl (2004), cuando se coloca la situación de vida por encima del ser, se imposibilita distinguir una de lo otro, es decir, la enfermedad se vive como totalidad y no como una parte de lo que es la persona. La vida ha perdido su valor y cuanto más depresiva se siente la persona, menos posibilidades de cambio ve o cree tener aún. No generaliza su experiencia, es decir, lo que vive no vale necesariamente para los demás. Por el contrario, la comparación con los demás se convierte en un motivo adicional de agudización de la depresión. No tiene la capacidad para comprender a los otros; vive en una constante perturbación y desorientación, en un tiempo sin tiempo, teme el día de mañana, se siente incapaz de poder soportar más, no le duele el hecho, no le duele la vida, le duele su vida.

Esta actitud íntima y profundamente sentida respecto a la vida es la respuesta a una suma de percepciones o, con mayor exactitud, de sensaciones relativas a la calidad de vida. La vivencia es, ante todo, un sentir, la manera en que se viven las propias experiencias, lo que se siente en esas vivencias es lo que proporciona la información sobre el valor de la vida. Si se repiten experiencias que representan la vida como algo sin valor, la vida misma se siente cada vez más sin valor y de aquí puede resultar una actitud responsiva negativa, Lukas (2001) plantea que el problema del balance de vida negativo no es la bondad de una vida vivida. Tampoco es la libertad de la vida que queda por vivir, sino lo malo que se ha convertido en destino, es decir, el mal sufrido y el sentimiento de culpa. Tragedias brutales, injustas e incomprensibles, y errores que nunca más se volverán a corregir. Así pues, las personas depresivas manifiestan un valor fundamental negativo (Längle, 2000). Por eso su concepción de la vida se torna en ser así y no poder ser de otra manera. Huyen de su responsabilidad, se fugan de sí mismos y se refugian en lo impersonal.

García (2003) señala en torno a la depresión en el adulto mayor, que por una parte éste acepta la irreversibilidad de la vejez, pero al mismo tiempo tiene claro que nada puede hacerse, lo que lleva al individuo a deprimirse. Esto es un paso necesario y satisfactorio en el proceso de entender su nueva situación y elaborar su condición de viejo, pues está en la búsqueda de su nueva identidad. Sin embargo, la fijación en esta etapa producirá una vejez signada por el desánimo y la melancolía, donde la vivencia del vacío interior se habrá instalado, favoreciendo la emergencia de síntomas asociados con la depresión.

Con base en la información revisada y dados los efectos y las reacciones típicas de la depresión, y ya que la etapa del adulto mayor se caracteriza por un sinfín de pérdidas que abarcan el plano psicofísico y social de 
la persona, es que consideró pertinente llevar a cabo este estudio.

\section{MÉTODO}

La investigación fue de corte cuantitativo y el estudio, de tipo comparativo. Se tuvo como objetivo general comparar el nivel de depresión en adultos mayores residentes del Valle de Toluca, a partir de las dimensiones que integran la Escala de Automedición de la Depresión de Zung, respecto a: sexo, edad, condición de retiro, actividad física y condición de salud. Se estableció como hipótesis de trabajo que la depresión que presentan los adultos mayores difiere de acuerdo con sus características sociodemográficas, tales como: el sexo, edad, condición de retiro, actividad física y condición de salud.

\section{Participantes}

Se trabajó con una muestra no probabilística de tipo intencional, integrada por 690 adultos mayores residentes del Valle de Toluca, México, de los cuales $51.16 \%$ fue de mujeres y $48.84 \%$ varones; $67.98 \%$ con edades entre 60-64 años y $32.02 \%$ entre $65-70$ años, $9.13 \%$ son solteros, $62.60 \%$ casados y $28.26 \%$ viudos.

\section{Instrumento}

La depresión fue medida con la Escala de Automedición de la Depresión, de Zung (1965), la cual permite establecer los síntomas físicos sin base orgánica aparente, además de medir las llamadas depresiones ocultas, muy comunes en la etapa del adulto mayor. Consta de 20 ítems, con cuatro opciones de respuesta ("nunca o muy pocas veces", "algunas veces", "frecuentemente", "la mayoría de las veces"). Mercado (2011) trabajó la confiabilidad y la validez de la escala en una muestra mexicana de adultos mayores, la varianza explicada fue de $45.582 \%$ y el alfa total de 0.852 . Para ello, se utilizó el análisis factorial exploratorio con rotación ortogonal (Varimax). El índice кмо fue de 0.896 , con una probabilidad $\leq 0.001$. Se conservaron los 20 ítems del instrumento original, que de acuerdo con la carga factorial se agruparon en tres dimensiones: 1) Fisiológica. Mide síntomas físicos relacionados con la depresión, como: variaciones del sueño, apetito, peso; problemas sexuales, gastrointestinales y cardiovasculares $(\alpha=0.818$ con
10 reactivos). 2) Psicológica. Evalúa intranquilidad, confusión, desesperanza, indecisión, irritabilidad, disgusto, ideas suicidas ( $\alpha=0.805$ con seis reactivos). 3 ) Insatisfacción personal. Se refiere a sensación de vacío y desvalorización personal, ideas suicidas ( $\alpha=0.635$ con cuatro reactivos).

\section{Procedimiento}

Se contactaron instituciones dedicadas a la atención y cuidado de adultos mayores, tales como IMSS, ISSEMYM, DIF, clubes de la tercera edad, así como centros religiosos, mercados, plazas públicas, ubicados en los municipios que conforman el Valle de Toluca (Toluca, Metepec, Lerma, San Mateo Atenco, Almoloya de Juárez y Zinacantepec). Una vez que se ubicadas las personas adultas mayores, se les aplicó la Escala de Automedición de la Depresión, así como la ficha de identificación de las variables demográficas. Se les informó sobre los aspectos generales y el objetivo de la investigación. Se solicitó su autorización por escrito (consentimiento informado) para participar en la presente investigación, y se les garantizó la confidencialidad de la información que proporcionaron y la utilización de ésta con fines de investigación.

Para establecer la comparación se utilizó la prueba t de Student para grupos independientes, la cual permitió observar diferencias entre las dimensiones que integran la escala en cuanto a sexo, edad, condición de retiro, práctica de actividad laboral y condición de salud. La prueba de hipótesis se efectuó con un nivel de significancia de 0.05 . Los datos fueron procesados en el programa estadístico sPss.

\section{RESULTADOS}

Se encontraron diferencias significativas en relación con el sexo, las mujeres (353) mostraron mayor presencia significativa de síntomas fisiológicos de depresión, a diferencia de los hombres (337). Respecto a la edad, los adultos mayores entre 65 y 70 años presentaron mayores síntomas fisiológicos de depresión que los de 60 a 64 años. En la condición de retiro, se tuvo que las personas no jubiladas presentan más síntomas fisiológicos de depresión que los jubilados (vid. tabla 1).

En cuanto a la actividad física, se pudo observar que los adultos mayores que no practican actividad fí- 
sica mostraron síntomas de depresión en las dimensiones fisiológica, psicológica y de insatisfacción personal, a diferencia de quienes si la practican (vid. tabla 2).

Respecto a la condición de salud, es decir, si se cursa o no con un padecimiento, los resultados obtenidos señalan que los adultos mayores que sí reportaron lo anterior mostraron puntajes mayores en cuanto a la presencia de síntomas depresivos de tipo fisiológico, psicológico y de insatisfacción personal (vid. tabla 3).

\section{DISCUSIÓN}

A partir del análisis de los resultados obtenidos en este estudio se rechaza la hipótesis nula, ya que se encontraron diferencias entre las dimensiones que conforman

Tabla 1. Prueba t de Student: diferencias por sexo, edad y condición de retiro en las dimensiones de la EAMD

\begin{tabular}{|c|c|c|c|c|}
\hline \multicolumn{3}{|c|}{ Dimensión fisiológica } & $t$ & Prob. \\
\hline Sexo & $\begin{array}{l}\text { Mujeres } \\
\text { Hombres }\end{array}$ & $\begin{array}{cc}M & 1.78 \\
D E & 0.57 \\
M & 1.68 \\
\text { DE } & 0.54\end{array}$ & 2.17 & 0.030 \\
\hline Edad & $\begin{array}{l}\text { 60-64 años } \\
65-70 \text { años }\end{array}$ & $\begin{array}{ll}M & 1.68 \\
D E & 0.54 \\
M & 1.84 \\
D E & 0.58\end{array}$ & 3.42 & 0.001 \\
\hline Condición de retiro & $\begin{array}{l}\text { Jubilado } \\
\text { No jubilado }\end{array}$ & $\begin{array}{cc}M & 1.63 \\
D E & 0.52 \\
M & 1.77 \\
\text { DE } & 0.57\end{array}$ & 2.95 & 0.003 \\
\hline
\end{tabular}

Tabla 2. Prueba t de Student: diferencias en cuanto a la práctica de actividad física en las dimensiones de la EAMD

\begin{tabular}{l|c|c|c|c|c|c}
\hline & \multicolumn{2}{|c|}{ No practican } & \multicolumn{2}{c}{ Sí practican } & \multicolumn{2}{c}{} \\
\hline Dimensión & $M$ & $D E$ & $M$ & $D E$ & $t$ & $P$ \\
\hline Fisiológica & 1.81 & 0.56 & 1.65 & 0.54 & 3.78 & 0.000 \\
\hline Psicológica & 2.09 & 0.70 & 1.90 & 0.71 & 3.53 & 0.000 \\
\hline Insatisfacción personal & 2.73 & 0.74 & 2.49 & 0.76 & 4.18 & 0.000 \\
\hline
\end{tabular}

Tabla 3. Prueba t de Student: diferencias en torno a la condición de salud (si se cursa o no con una enfermedad) en las dimensiones de la EAMD

\begin{tabular}{|c|c|c|c|c|c|c|}
\hline \multirow[b]{2}{*}{ Dimensión } & \multicolumn{2}{|c|}{ No } & \multicolumn{2}{|c|}{ Sí } & \multirow[b]{2}{*}{$\mathrm{t}$} & \multirow[b]{2}{*}{$P$} \\
\hline & $M$ & $\mathrm{DE}$ & M & $\mathrm{DE}$ & & \\
\hline Fisiológica & 1.62 & 0.53 & 1.78 & 0.56 & 3.46 & 0.001 \\
\hline Psicológica & 1.96 & 0.72 & 2.04 & 0.70 & 2.01 & 0.040 \\
\hline Insatisfacción personal & 2.45 & 0.76 & 2.69 & 0.74 & 3.90 & 0.000 \\
\hline
\end{tabular}


la Escala de Automedición de la Depresión en relación con el sexo, la edad, condición de retiro, actividad física y condición de salud.

En cuanto al sexo de los participantes, las mujeres mostraron mayor tendencia a presentar síntomas fisiológicos a diferencia de los hombres; lo cual coincide con lo que afirma Ortiz de la Huerta (2004), quien refiere que las mujeres viven tres o cuatro años más que los hombres, por lo cual no mueren a causa de la vejez, sino por las enfermedades que acompañan a ésta. Este grupo, por tanto, presenta menor mortalidad, pero mayor morbilidad, es decir, las mujeres se enferman más, pero no mueren en seguida, sino que duran mucho tiempo con padecimientos que, por lo general, son crónico-degenerativos.

Respecto a la edad, los adultos mayores con edades entre 65 y 70 años refieren una mayor presencia de síntomas depresivos de tipo fisiológico, lo cual coincide con lo que afirman Mishara y Riedel (2000), quienes refieren que los cambios fisiológicos que se producen a partir de la sexta década de la vida comportan una complejidad que pueden predisponer la aparición de varias enfermedades en forma simultánea. Al presentarse juntas dichas dolencias, se potencian entre sí.

En lo que se refiere a la condición de retiro se encontró que las personas que no tienen el estatus de jubilados tienden más a mostrar síntomas fisiológicos de depresión. De acuerdo con García (2003), la forma de percibir la condición de retiro dependerá, en gran medida, de factores personales y económicos, es decir, una persona optimista ante la vida, que goce de buena salud y no tenga preocupaciones económicas podrá afrontar su retiro laboral con mayor éxito en la adaptación a su nueva situación sociofamiliar. Cuando se tiene una situación económica precaria y mala salud, la jubilación constituirá una carga difícil de superar.

Un factor de protección en la vejez es que la persona sea capaz de realizar actividades sustitutivas, que coadyuven a mantener un alto sentido de satisfacción con la vida. Ello repercutirá en la visión que tenga la persona de sí misma y de su situación de vida. Cabe aquí señalar que un aspecto que favorece el envejecimiento psicológico es la capacidad de adaptación para afrontar los avatares del envejecimiento. Lo anterior concuerda con los resultados obtenidos, los cuales mos- traron que los adultos mayores que no realizan actividad física tienden a presentar síntomas fisiológicos, psicológicos y de insatisfacción personal vinculados con la depresión. Al respecto, Frankl (2001) explica que cuando el hombre se niega a sí mismo, vive a merced de la facticidad de su destino, dominado por aquello que compete al plano psicofísico, no puede distanciarse de sí mismo, pues se llena de vacío, desesperación y de sufrimiento innecesario, al no poder articular su capacidad de oposición frente a los condicionamientos no sólo biológicos, sino también psicológicos o sociales; por tanto, se encuentra determinado por dichos condicionamientos, que van asociados con alguna enfermedad.

Con base en los resultados obtenidos, las personas que cursan con algún padecimiento mostraron una clara tendencia a presentar síntomas depresivos en las dimensiones fisiológica y psicológica, así como de insatisfacción personal; Frankl (2004) explica que cuando se coloca la situación de vida por encima del ser, se imposibilita distinguir una de la otra, es decir, la enfermedad se vive como totalidad y no como una parte de lo que es la persona, esto conduce, de acuerdo con Acevedo y Battafarano (2003), a no poner distancia entre lo que se tiene (enfermedad) y lo que se es (persona). En el transcurso de su vida el ser humano pasa por distintas etapas de desarrollo, signadas por crisis existenciales, propias de los procesos de adaptación a los requerimientos resultantes de los distintos roles a asumir. En palabras de Gallo y Kasses (2009), de todas estas crisis, sin lugar a dudas, dentro de las más conflictivas está la vejez. El pasaje a la vejez presenta una situación simétricamente opuesta de desesperanza, pues conlleva perder posiciones alcanzadas con mucho esfuerzo, para retroceder en el estatus social, abandonar roles productivos e incorporarse a la población pasiva. $\mathrm{El}$ individuo ya no elige su futuro, sino que se prepara para su muerte, primero social y más adelante, física. En esta difícil crisis vital, el individuo debe prepararse para asumir la irrenunciable responsabilidad de darle una respuesta con sentido a esta nueva invitación a la vida que significa la propuesta de una nueva edad. Este desafío no es fácil, pues se está inmerso en una cultura que sobrevalora la vida plena, la juventud, la sexualidad genital y orgásmica, la rapidez y la productividad. • 


\section{REFERENCIAS}

Acevedo, G. \& Battafarano, M. (2003). Desde Viktor Frankl. Hacia un enfoque transdisciplinario del enfermar humano. Buenos Aires: FAL.

Arriola, E. (2000). La edad de la vida: hacia un envejecimiento saludable y competente. San Sebastián: Erein.

Ávila-Funes, J., Melano-Carranza, E., Payette, H. \& Amieva, H. (2007). Síntomas depresivos como factor de riesgo de dependencia en adultos mayores. Salud Pública de México, 49(5), 367-375. Recuperado de http://www.scielo.org.mx/scielo.php?script=sci_artt ext\&pid=S00366342007000500007\&lng=es

Blazer, D. G. (2003). Depression in late life: review and commentary. J Gerontol A Biol Sci Med Sci, 58, 249-265.

Consejo Nacional de Evaluación dela Política de Desarrollo Social (Coneval) (2012). Informe de Evaluación de la Política de Desarrollo Social. Recuperado de http://www.coneval.gob.mx/Informes/Evaluacion/ IEPDS2012/Pages-IEPDSMex2012-12novVFinal_ lowres6.pdf

Encuesta Nacional sobre Salud y Envejecimiento en México (Enasem) (2012). Estudio Nacional de Salud y Envejecimiento en México. Recuperado de http: //www.inegi.org.mx/inegi/contenidos/espanol/ prensa/Boletines/Boletin/Comunicados/Especiales/ 2013/septiembre/comunica14.pdf

Fizzotti, E. (2006). De Freud a Frankl: el nacimiento de la logoterapia. Guía teórico-práctica para quien busca el sentido de la vida. México: LAG.

Frankl, V. (2001). Ante el vacío existencial. Hacia una humanización de la psicoterapia (8a. ed.). Barcelona: Herder.

Frankl, V. (2004). El hombre en busca de sentido. Barcelona: Herder.

Gallo, P. \& Kasses, P. (2009). Contribuciones para un mejor tránsito a la tercera edad. Recuperado de http:// www.rediberoamericanadetrabajoconfamilia.org.
García, C. (2003). Cita a ciegas. Buenos Aires: San Pablo. Iglesias, J. (2001). La soledad de las personas mayores. Influencias personales, familiares y sociales. Análisis cualitativo. Madrid: Imserso.

Instituto Nacional de Estadística y Geografía (INEGI) (2010). Censo de Población y Vivienda 2010. México: Instituto Nacional de Estadística y Geografía.

Längle, A. (2000). Depression [Depresión]. En G. Stumm, A. Pritz (Eds.). Wörrterbuch der Psychoterapioe [Diccionario de psicoterapia]. Wien: Springer.

Längle, A. (2004). Psicoterapia analítico-existencial de los trastornos depresivos. Neuropsiquiatria, 42(3), julio, 195-206.

Lukas, E. (2001). Sobre la transitoriedad de la vida. En E. Lukas y C. l. García (Eds.). De la vida fugaz. Buenos Aires: Almagesto.

Mercado, A. (2011). Devenir existencial del adulto mayor (Tesis doctoral inédita). Universidad Iberoamericana, Campus Santa Fe, México.

Mishara, B. T. \& Riedel, R. G. (2000). El proceso de envejecer (3a. ed.). Madrid: Morata.

Ortiz de la Huerta, D. (2004). Aspectos sociales del envejecimiento. México: Archivo Geriátrico. Asociación Mexicana de Gerontología y Geriatría.

Pederzini, C. (2010). Envejecimiento demográfico en México, principales causas y características. En R. Gutiérrez, M. Picardi, N. Aguilar, F. Ávila, J. Méndez y A. Pérez. Gerontología y nutrición en el adulto mayor. México: McGraw-Hill Interamericana.

Peláez, M. (2005). La construcción de las bases de la buena salud en la vejez: situación en las Américas. Revista Panamericana de Salud Pública 17, 299-302.

Salvarezza, L. (2005). Psicogeriatría. Teoría y clínica. Buenos Aires: Paidós.

Zung, W. W. K. (1965). A self-rating depression scale. Archives of General Psychiatry, 12, 63-70. 